\title{
Increased respiratory burst and phosphodiesterase activity in alveolar eosinophils in chronic eosinophilic pneumonia
}

\author{
R. Aloui*, F. Gormand*+, A.F. Prigent*, M. Perrin-Fayolle+, Y. Pacheco+
}

\begin{abstract}
Increased respiratory burst and phosphodiesterase activity in alveolar eosinophils in chronic eosinophilic pneumonia. R. Aloui, F. Gormand, A.F. Prigent, M. Perrin-Fayolle, Y. Pacheco. @ ERS Journals Ltd 1996.

ABSTRACT: A 43 year old woman presented with chronic eosinophilic pneumonia characterised by a high alveolar eosinophilic count, which allowed biochemical study of these cells.

Alveolar eosinophils spontaneously produced high amounts of oxygen free radicals and exhibited an increased level of cyclic adenosine monophosphate (cAMP) phosphodiesterase (PDE) activity compared to blood eosinophils from control or allergic subjects. This activity was preferentially located in the plasma membrane, whilst the PDE activity of blood eosinophils from asthmatics or controls predominated in the cytosol.

Because of the potential role of phosphodiesterase during eosinophil activation and recruitment, phosphodiesterase inhibitors may be useful in the treatment of eosinophilic pneumonia.
\end{abstract}

Eur Respir J., 1996, 9, 377-379.

+Service de Pneumologie, Centre Hospitalier Lyon-Sud, France. *INSERM unité 352, Villeurbanne, France.

Correspondence: Y. Pacheco

Service de Pneumologie

Centre Hospitalier Lyon-Sud

69310 Pierre Bénite

France

Keywords: Eosinophilic pneumonia phosphodiesterase respiratory burst

Received: March 151995

Accepted after revision August 301995
Chronic eosinophilic pneumonia (CEP) is characterized by a high eosinophil count in bronchoalveolar lavage (BAL) fluid, severe constitutional symptoms, pulmonary infiltrates predominantly in the upper lobes, and a good response to steroid therapy $[1,2]$. Morphological, biochemical and functional studies of eosinophils from blood and tissues of patients with eosinophil-related diseases have been reported [3]. Cyclic nucleotides and enzymes linked to their metabolism, such as cyclases or phosphodiesterases (PDE), are involved in cell activation and differentiation. We have previously shown an increased cyclic adenosine monophosphate (cAMP) PDE activity in blood leucocytes from allergic subjects [4]. At least five different PDE isoenzyme families exist. Among them, the type IV appears to be the predominant isoenzyme catabolizing cAMP in leucocytes [5]. Recently, Newsholme and Schwartz [6] reported that rolipram, a PDE IV selective inhibitor, reduced eosinophil infiltration in conjunctiva from guinea-pigs.

We report a biochemical study of the PDE activity and the oxygen free radical generation in alveolar eosinophils collected from a patient presenting with CEP.

\section{Case report}

A 43 year old nonsmoking woman experienced three episodes of cough, fever, dyspnoea and weight loss between April 1977 and March 1978. On each occasion her chest roentgenograms revealed uni- or bilateral alveolar interstitial infiltrates of the upper lobes. Eosinophilic blood counts were increased (range 1,020-14,000 $\cdot$ cells $\cdot \mathrm{mm}^{-3}$ ). In March 1978, the cellular count of BAL revealed 53\% eosinophils. Microbiological studies were negative. The clinical diagnosis was cryptogenic eosinophilic pneumonia and the patient was treated each time with prednisone for 2-3 months. Infiltrates always cleared and the patient became asymptomatic.

Nine years later, the patient presented with cough, dyspnoea, fever and alveolar infiltrate of the left upper lobe. She had a peripheral eosinophil count of 3,300 cells $\cdot \mathrm{mm}^{-3}$. BAL was performed and a cell count revealed $1.09 \times 10^{9}$ cells $\cdot 100 \mathrm{~mL}^{-1}$, with $93 \%$ eosinophils. Her serum immunoglobulin E (IgE) level was normal. Tuberculin and fungal skin tests were nonreactive. Cultures for sputum and BAL were negative for bacterial, parasitic, mycobacterial and fungal organisms. Serologies for parasites and fungal organisms were also negative. Antinuclear antibodies, anti-deoxyribonucleic acid (DNA) antibodies and rheumatoid factor were not detected. Results of roentgenogram of the sinuses, and urinanalysis were normal. Analysis of arterial blood in room air revealed a $\mathrm{pH}$ of 7.48 an arterial oxygen tension $\left(\mathrm{Pa}, \mathrm{O}_{2}\right)$ of 10.1 $\mathrm{kPa}$ and an arterial carbon dioxide tension $\left(\mathrm{Pa}_{\mathrm{a}} \mathrm{CO}_{2}\right)$ of 4 $\mathrm{kPa}$. The transfer factor of the lungs for carbon monoxide was $105 \%$ of the predicted value. The patient was treated with prednisone for 7 weeks. At this time, all symptoms and roentgenogram infiltrates had disappeared. Her $\mathrm{Pa}, \mathrm{O}_{2}$ was $13 \mathrm{kPa}$. Since 1987, no relapse has occurred.

\section{Materials and methods}

Oxygen free radical generation and cyclic nucleotide phosphodiesterase activity were studied in alveolar 
eosinophils collected in this patient. Data were compared with values obtained from blood eosinophils of control subjects or asthmatic patients.

\section{Subjects}

Five mild allergic asthmatics ( 3 females and 2 males, mean age $34 \mathrm{yrs}$ ) had positive allergic skin tests and elevated specific IgE levels. Asthma was defined by a previous history of episodic wheezing and reversible airway obstruction. Antiasthma treatment included $\beta$-agonist alone and was stopped $8 \mathrm{~h}$ before blood punction.

Seven nonsmoking healthy volunteers, with a negative history of atopy or other disease, served as controls (4 females and 3 males, mean age 39 yrs).

\section{Cell preparations}

Venous blood eosinophils from controls or allergic asthmatics were isolated as described previously [7]. In the eosinophilic pneumonia alveolar eosinophil purification was not needed, since BAL contained $93 \%$ of eosinophils. To separate into cytosolic and particulate components, the eosinophil homogenate was centrifuged at $105,000 \times \mathrm{g}$. The resulting supernatant was used as a source of cytosolic PDE activity. Pellet fractions rehomogenized in Tris-HC1 buffer were used as a source of particulate PDE activity.

\section{Oxygen free radical generation}

Oxygen free radical generation by eosinophils, without any antigen challenge, was measured in a Biocounter Lumac liquid scintillation counter and was expressed as related light international units (RLIU) per $10^{5}$ cells.

\section{Phosphodiesterase assays}

The AMP PDE activity was assayed according to a modified method based on the original two step radioisotopic procedure of THOMPSON et al. [8], with $0.25 \mu \mathrm{M}$ cyclic nucleotide as substrate. Assays were performed either with crude homogenates, cytosolic fractions or cell membrane fractions. PDE activity was expressed as pmol cyclic nucleotide hydrolysed $\cdot \mathrm{min}^{-1} \cdot \mathrm{mg}$ protein.

\section{Isoelectric focusing assay}

The PDE isoenzyme profile of the cytosolic fraction from BAL eosinophils was analysed by isoelectrofocusing on polyacrylamide gel plates. The method has been described in detail in a previous report [9].

\section{Results}

\section{Oxygen free radical generation}

Without any stimulation, alveolar eosinophils from the hypereosinophilic pneumonia produced a very high level of oxygen free radicals (265,210 RLIU per $10^{5}$ cells).
This value is higher than those obtained in blood eosinophils from control subjects $(6,603 \pm 3,029 ; n=5)$ or from asthmatics $(10,850 \pm 4,090 ; n=7)$.

\section{Cyclic AMP PDE activity}

Total cAMP PDE activity measured in the whole eosinophil homogenates was higher in the BAL eosinophils from the eosinophilic pneumonia $\left(18.63 \mathrm{pmol} \cdot \mathrm{min}^{-1} \cdot \mathrm{mg}\right.$ protein) than in blood eosinophils from the control group $(9.3 \pm 2.8)$ or asthmatic subjects $\left(13.0 \pm 3.1 \mathrm{pmol} \cdot \mathrm{min}^{-1} \cdot \mathrm{mg}\right)$. We did not observe any cyclic guanosine monophosphate (cAMP) PDE activity. In the control subjects, $78 \pm 12 \%$ of the cAMP PDE was recovered in the cytosolic fraction of blood eosinophils and $22 \pm 7 \%$ in the particulate fraction. Similar distribution of cAMP PDE activity was obtained in blood eosinophils from asthmatic subjects $(70 \pm 11$ and $30 \pm 6 \%)$ and in BAL eosinophils from an asthmatic patient (62\% in the cytosolic fraction). However, this distribution was different in BAL eosinophils from the eosinophilic pneumonia, where the PDE activity was essentially found in the membrane fraction of BAL eosinophils (90\%). Conversely, predominant localization of cAMP PDE in the cytosolic fraction was also shown in blood eosinophils of another patient with CEP (79\% in the cytosolic fraction).

Isoelectric focusing characterization of CAMP PDE isoenzymes

Isoelectric focusing analysis of the cytosolic fraction (fig. 1) revealed a single cAMP hydrolysing activity with an isoelectric point of 5.8. This activity was inhibited by the cAMP-PDE selective-inhibitor, rolipram (concentration inhibiting substrate hydrolysis by $50 \%\left(\mathrm{IC}_{50}\right)$ $=4 \times 10^{-6} \mathrm{M}$ ) (fig. 2). Unfortunately, characterization of the PDE activity in the membrane fraction failed, since this particulate activity could not be solubilized in the classic detergents, such as lubrol or sodium cholate.

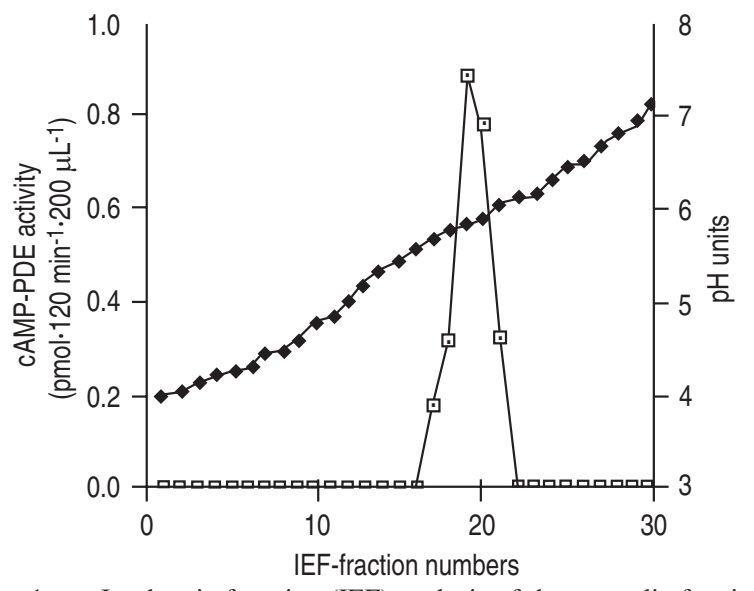

Fig. 1. - Isoelectric focusing (IEF) analysis of the cytosolic fraction of alveolar eosinophils. This technique revealed a single cAMP hydrolysing activity between fractions $16-22$. Isoelectric point was 5.8. No cGMP hydrolysing activity was detected in any fraction. $\square$ : PDE activity; $\bullet: \mathrm{pH}$ gradient. cAMP: cyclic adenosine monophosphate; cGMP: cyclic guanosine monophosphate; PDE: phosphodiesterase. 


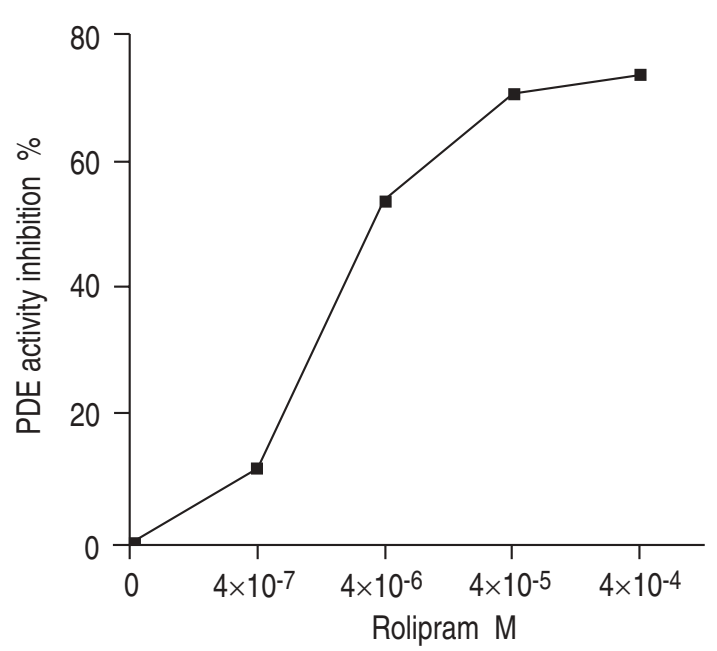

Fig. 2. - Inhibition of the cytosolic cAMP PDE activity of alveolar eosinophils by rolipram. The concentration that inhibited substrate hydrolysis by $50 \%$ (IC50) was $4 \times 10^{-6} \mathrm{M}$. IC50 value was determined from concentration-response curves. Results are representative of three determinations. For abbreviations see legend to figure 1.

\section{Discussion}

A major limitation to the characterization of eosinophil function in humans arises from difficulties in isolating these cells in high purity and bulk. In our patient with chronic eosinophilic pneumonia a striking feature was the purity and the very high number of eosinophils in BAL, which allowed biochemical study of these cells.

Generally, the active role of eosinophils in inflammation is believed to be due to the release of cytotoxic products, such as basic proteins, the synthesis of lipid mediators and the generation of reactive oxygen species [10]. In this study, BAL eosinophils had a very high spontaneous generation of superoxide anions. Products of oxidative metabolism which are one of the markers of eosinophil function are toxic to the pulmonary tissue contributing to the development of eosinophil-derived airway injury.

Cyclic AMP PDE has been studied in several inflammatory cells [11-13]. An increased cAMP PDE activity could contribute to the decrease of cAMP levels in neutrophils during their activation process [11]. We found an increased level of cAMP PDE activity in BAL eosinophils from a patient presenting with eosinophilic pneumonia as compared to blood eosinophils from control or allergic subjects, and we noted an absence of cGMP PDE in these cells. This activity was preferentially located in the membrane, whilst the PDE activity of blood eosinophils from asthmatics or controls predominated in cytosolic fractions. The PDE localization was determined in blood eosinophils from one case of CEP and in alveolar eosinophils from one asthmatic patient. In both cases, the PDE activity predominated in the cytosolic fraction.

Taken together, these results could suggest a particular localization of the PDE activity in the BAL eosinophils from our patient with CEP. Several hypotheses could be suggested to explain this last observation: 1) an eosinophil PDE membrane isoform which has not previously been described; 2) a translocation process of this activity from the cytosol to the membrane during the activation or the differentiation of eosinophils; 3) an abnormal PDE protein in eosinophilic pneumonia.

We attempted to characterize this PDE activity by the isoelectrofocusing method, since the eosinophilic pneumonia gave rise to a sufficient amount of cells to allow such a study. The characterization of PDE activity in the membrane fraction failed due to its high insolubility in classic detergents. Isoelectric focusing analysis of the cytosolic fraction, which contains only $10 \%$ of the whole PDE activity of BAL eosinophils, revealed a single cAMP hydrolysing activity which was sensitive to rolipram, a PDE IV specific inhibitor. No cGMP activity was detected in this fraction, thus suggesting that this activity is a PDE type IV. Because of the potential role of phosphodiesterase during eosinophil activation and recruitment, phosphodiesterase inhibitors may be useful in the treatment of eosinophilic pneumoniae.

\section{References}

1. Jederlinic JP, Sicilian L, Gaensler EA. Chronic eosinophilic pneumonia: a report of 19 cases and a review of the literature. Medicine 1988; 67: 154-162.

2. Naughton M, Fahy J, Fitzgerald MX. Chronic eosinophilic pneumonia: a long-term follow-up of 12 patients. Chest 1993; 103: 162-165.

3. Gleich GJ, Adolphson CR. The eosinophil leukocyte structure and function. Adv Immunol 1986; 39: 177-253.

4. Prigent AF, Fontlupt P, Dubois M, et al. Activity of cyclic AMP phosphodiesterase and methyltransferases in leukocyte membranes from allergic patients. Clin Chim Acta 1984; 143: 225-233.

5. Beavo J. Multiple phosphodiesterase isoenzymes: background, nomenclature and implications. In: Beavo J, Houslay MD, eds. Cyclic Nucleotide Phosphodiesterases: Structure, Regulation and Drug Action. New York, John Wiley and Son, 1990; pp. 3-15.

6. Newsholm SJ, Schwartz L. cAMP-specific phosphodiesterase inhibitor, rolipram, reduces eosinophil infiltration evoked by leukotrienes or by histamine in guinea-pig conjunctiva. Inflammation 1993; 17: 25-31.

7. Shult PA, Graziano FM, Busse WW. Enhanced eosinophil luminol-dependent chemiluminescence in allergic rhinitis. J Allergy Clin Immunol 1986; 77: 702-708.

8. Thompson WJ, Brooker G, Appleman MM. Assay of cyclic nucleotide phosphodiesterase with radioactive substrates. Methods Enzymol 1974; 38: 205-212.

9. Némoz G, Prigent AF, Pageaux JF, Pacheco H. Isoelectric focusing pattern of cyclic nucleotide phosphodiesterase from rat heart. Biochem J 1981; 119: 113-119.

10. Prin L, Capron M, Gosset P, et al. Eosinophilic lung disease: immunological studies of blood and alveolar eosinophils. Clin Exp Immunol 1986; 63: 249-257.

11. Grady PG, Thomas LL. Characterization of cyclicnucleotide phosphodiesterase activities in resting and $\mathrm{N}$ formylmethionyl-leucylphenylalanine-stimulated human neutrophils. Biochim Biophys Acta 1986; 885: 282-293.

12. Chan SC, Reifsnyder D, Beavo JA, Hanifin JM. Immunochemical characterization of the distinct monocyte cyclic AMP-phosphodiesterase from patients with atopic dermatitis. J Allergy Clin Immunol 1993; 91: 1179-1188.

13. Epstein PM, Hachisu R. Cyclic nucleotide phosphodiesterase in normal and leukemic human lymphocytes and lymphoblasts. Adv Cyclic Nucleotide Res 1984; 16: 303-321. 
